\title{
Innovative strategy for the pharma of the future: summary of trends and projections 2017-2018
}

\begin{abstract}
The Pharmaceutical industry contributes significantly both nationally and globally to healthcare development, economy, and technology. According to the annual World Preview report 2016, ${ }^{1}$ the global pharmaceutical market will reach $\$ 1.12$ trillion in 2022 with a stable growth rate of $6.3 \%$ CAGR. Even so, the successful future of this industry might be at risk due to the growing political uncertainties, rising skepticism over the drugs pricing, reimbursement approaches, and mounting need to understand and adopt new technologies. ${ }^{2}$ Existing business models where R\&D, commercial, production, and supply chain components act in silos as unrelated modules, often disconnected from the external partners in regulatory, policymaking, and medical worlds. These business models can no longer sustain the pressure and requirements of the modern market. ${ }^{3}$ To understand and monitor behavior of this industry and its impact on National and Global economy, the consulting firms prepare comprehensive annual reports dedicated explicitly to the upcoming trends in drug development industry. Recommendations are made to reevaluate existing business models and to reshape them with cross-functional strategies in order to overcome existing industry external and internal obstacles. In addition to meticulously prepared annual reports, individual experts in the field use Social Media venues to continuously contribute to the formation of public opinion about Pharma trends and policies by expressing their thoughts and ideas online. In this review we summarized twenty-four reports, articles, and posts with projections and trends in pharmaceutical industry for years 2017 and 2018. The focus of this review is on two major requirements for the Pharmaceutical industry of the future: innovative corporate strategy, and technology strategy.
\end{abstract}

Keywords: pharma, pharmaceutical, innovative, strategy, drug development, healthcare, high-tech, technology, trends, projections
Volume 7 Issue 2 - 2018

\author{
Irina I Agoulnik \\ CEO,Vesti Intelligence Corp, USA
}

Correspondence: Irina I Agoulnik, CEO, Vesti Intelligence Corp., 67 S. Bedford St, STE 400W, Burlington, MA 0182I, USA, Tel 978-684-28I2, Email i.agoulnik@vesticorp.com

Received: March 05, 2018 | Published: March 19, 2018
Abbreviations: AI, artificial intelligence; EY, EY (formerly Ernst \& Young), a multinational professional services firm, London, England; CAGR, compound annual growth rate; CAR-T, chimeric antigen receptor; chimeric $\mathrm{T}$ cell receptor; or artificial $\mathrm{T}$ cell receptor; CRISPR, clustered regularly interspaced short palindromic repeats; FDA, food and drug administration; GE, general electric ventures; GMP, good manufacturing practice; HIPAA, health insurance portability and accountability Act of 1996; HITECH, health information technology for economic and clinical health act; IBM, international business machines corporation; IoT, internet of things; M\&A, mergers \& acquisitions; NME, new molecular entity; $\mathrm{R} \& \mathrm{D}$, research and development

\section{Introduction}

Traditionally, strategic planning in Pharma industry focuses on four major parts of the corporate management: regulatory affaires, pricing and market access, government affairs, and medical affairs. This approach assures a cross-organizational compliance and collaboration in evidence generation and management $(R \& D$, pharmacovigilance), in customer management (sales, marketing, key accounts), and in supply chain maintenance. Typical analyses of trends in drug development industries estimate the upcoming changes and the potential impact these changes might have on the industry going forward. For example, it was projected that in year 2017 the homecare sector will grow, that truly personalized medicine for chronic and other diseases will step up in development, and that current drug development pipelines with complex molecules will continue to drive the evolution of new manufacturing techniques. In addition, the forces of globalization will require industry players to adapt to strong demographic, political and technological shifts in order to successfully compete on the market. ${ }^{4-8}$ The Pharma corporate strategy typically includes global politics and healthcare policies, choice of therapeutic domains, partnerships and alliances, technology and drug manufacturing. Nowadays however, the technological advances that rapidly change industry's landscape, do call urgently for creation of a new section in corporate strategy - High-Tech and Internet strategy, or Technology strategy. Extensive use of Internet for communication, marketing, and customer engagement, as well as annual generation of massive scientific and clinical datasets brings to focus Big Data analytics and Artificial Intelligence strategies. Moreover, adoption of new bioengineering and high-tech technologies for drug development offers another exciting strategy path as it opens the whole new world of opportunities for the Pharma industry. ${ }^{9,10}$ Here, we emphasize the growing need in drug development industry to reshape the Corporate Strategy model to include a special sector - Technology Strategy with designated executive oversight. While being designed as unique, innovative and comprehensive, the new model for Corporate Strategy should speak to the needs of the market, the customer, and ultimately - the needs of patients. 


\section{Innovative corporate strategy}

Both international and national political milieus play substantial roles in global economic development and as such affect every industry, including the pharmaceutical one. Brexit vote (2016), for example, has a multilayered effect on European pharmaceutical companies forcing them to revisit their existing initiatives and to generate new ideas (e.g. support small companies). ${ }^{11}$ The impact of the Trump effect on Pharma industry and healthcare (2017) is still to be fully assessed, but can already be seen via drug prescription 'lenses': millions of lost health insurances that cover prescription drugs, would mean fewer customers and less revenue for pharmaceutical companies. Furthermore, the healthcare policy-makers and payers become more cautious about truly innovative medicines. They also begin to play a bigger role in prescription regulations by establishing sustainable compliance across the board with tighter regulatory guidelines for drug labeling, such as global serialization., ${ }^{5,12,13}$ On the other hand, global population ageing, evolution of consumer spending and global economy continuously creates new requirements for medicines. Not surprisingly then that demands for medicines grow at a much larger and faster rate in the emerging economies than in the industrialized ones. Some governments switch also their focus from disease treatment to disease prevention, which creates an additional drive for pre-emptive measures investments. Each of these factors may have an effect on corporate strategy design depending on company's mission and goals.

Current shifts in healthcare policies are caused in part by the fact that different forms of the industry are now merging as clinical advances transform previously fatal diseases into the chronic ones and self-medication sectors started to grow. Additionally, patients are increasingly empowered by the option to choose the prescriptions they purchase or the treatment they receive. As a result, the significance of patients' personal education, perception of their illnesses, and the value of treatments they receive, present a new serious challenge for Pharma industries. These are not the only factors that can be addressed by innovative corporate strategy design. Partnerships and alliances also require a closer look and consideration going forward. Bigger mergers and acquisitions, movements toward more equitable attitudes, and collaborations in the digital sector are all good indicators of the growing need in strategic partnerships. ${ }^{14}$ An aggressive invasion of High-tech into the Pharma sector (e.g. pills that track patient compliance or inhalers that monitor when each dose is taken) creates new types of partnership with a true competitive edge. Ever-growing high-tech demands (Internet Strategy, Artificial Intelligence) and the growth of the homecare sector treatments (e.g. self-administered drugs, pre-filled syringes, etc.) require intricate partnership management systems. ${ }^{15}$ This is a high call for designing the strategy for the Pharma of the Future, but challenges hardly end here. To build a unique, company-specific strategy the management team would need to address a number of already known industry challenges such as ambiguity, rising customer expectations, scientific productivity, and "cultural sclerosis"., Analysts recommend a combination of approaches for new strategy design, such as extended value demonstration, streamlined and flexible supply chain, sharp growth choices, personalized medicine capability systems, global footprint, and optimized resources. While global politics and healthcare policies outline market environmental demands, stringencies, and opportunities, the newly designed corporate strategy will have to meet the local market demands first.
Drug discovery modernization has historically been the driver of industry growth, yet currently it may suffer from limited savings due to shifting usage to generics, as big, blockbuster drugs patents (\$17B worth) are expected to expire over the next ten years. ${ }^{16,17}$ To compensate for losses, big companies increase their M\&A activity. Here, investors are forewarned to look for companies with healthy pipelines of drug development for "orphan" diseases, and to be aware of the growth in Asian markets as "China currently accounts for a quarter of the global healthcare market." Nevertheless, the innovation in drug discovery and development is still the key in the ever changing Pharma landscape, since the big decrease in the number of drug approvals by FDA in US [from 56 (2015) to 27 (2016)], came back to 46 FDA-approved drugs in $2017 .{ }^{18}$

The cost of drug development and manufacturing grow continuously while the outsourcing drives the accelerated growth of R\&D. To recover the costs and still be profitable, some companies implemented fixed annual or quarterly drug price increases to fuel sales growth. The proposed initiative involving efficient waste and defects removal at the manufacturing facilities has a potential to reduce the cost of the produced goods, but not by much. It was indicated however, that the only proper way to create sustainable revenue is through manufacturing volume. ${ }^{5}$ To achieve more efficient manufacturing operations the end-to-end integrated supply chain business solutions are implemented, and manufacturing network is continuously realigned.

Moreover, the traditionally conservative healthcare ecosystem is also getting restructured: a) the business development surprises market with deals where payors buying providers (UnitedHealthcare, via Optum subsidiary) and e-commerce giants (Amazon) considering entering Pharma space, ${ }^{19} \mathrm{~b}$ ) the widespread use of electronic medical records gradually provides sufficient data for outcomes-based pricing creation, and c) the improved regulatory stability in some sectors of healthcare ecosystem allow to focus on cost containment (e.g. payors), and industry consolidation.

Despite ongoing modifications and associated instability, the Pharma industry is staying strong. The volume of deals in 2017 was the highest in biotech industry since 2010 (471, \%9.3B), and the venture-backed funding for deals in 2018 will likely be up from that benchmark. ${ }^{20}$ The worldwide pharmaceutical R\&D spend is also growing up to $\$ 181$ bn in 2022 with $2.4 \%$ CAGR, while an average R\&D spend per NME stays at $\$ 4$ bn over the last 10 years. ${ }^{18}$

Driven by the need to overcome external and internal challenges, the simultaneous multi-layered transformation of the industry demands new business approaches and fresh models for corporate strategy. Designing innovative corporate strategy is tedious yet exciting task, the one that positions organization advantageously for the future successful operations and fulfillment of obligations to patients and shareholders.

\section{Technology strategy}

Technological advances affect every corner of our lives. Not surprisingly then that the Pharma industry is being affected by them too. While traditional drug manufacturing improvements (continuous and lean manufacturing, Six Sigma methodology, better planning tools, GMP standards at third-party contract manufacturers and suppliers, etc.), do bring manufacturing to the next level, the IoT takes a robust path toward streamlining product development cycle. Another bold 
application of technology in manufacturing is $3 \mathrm{D}$-prining ${ }^{15}$ with the first FDA-approved drug SPRITAM ${ }^{\circledR}$ (levetiracetam) developed by Aprecia Pharmaceuticals as an adjunctive therapy for the treatment of epilepsy.

Ground-breaking technologies in molecular engineering (CAR-T) and gene editing (CRISPR-CAS9) are expected to take over the traditional drug discovery and development approach, while brining new hope to patients and redefining drug discovery as we know it. The Pharma industry nowadays is focused on developing personalized medicine for chronic and other diseases with strong market demands in drug development for neurodegenerative diseases (Alzheimer's in particular), the microbiome, vaccines for the developing world and beyond, and treatments in competitive immuno-oncology domain. Oncology is identified as a continuous frontrunner in drug development pipeline with a total of 137 drug candidates followed by drugs in pain (40), and central nervous system (30) in Pharmaceutical industry. ${ }^{16-20}$

Recent outstanding breakthrough in drug discovery sector is accompanied by a strong shift towards Big Data analysis for BioPharma businesses and R\&D. Big Data analysis brings promises of unsurpassed benefits of innovative solutions from deep learning and artificial intelligence (AI). The complexity of digital disruption extends inevitably to new technical and behavior-driven challenges for Pharma such as data integrity, data types, data quality, and operational issues. In addition to traditional clinical laboratory results and multi-omics analysis data, new data types are being generated and used by the upstream technologies, such as real-time data from wearables and other mobile technologies, behavioral data collected from social media sites (e.g., Facebook and Twitter) and advocacy organizations (e.g., PatientsLikeMe). ${ }^{21}$ Moreover, as industry embraces new products for technically empowered patients such as augmented and virtual reality, body sensors, and activity monitors ${ }^{15}$ it has to pay much closer attention to regulations and recent changes in HIPAA and HITECH. ${ }^{22}$

Reportedly, the majority of the healthcare industry executives are preparing to embrace AI by 2025 . Yet, there is still very little evidence of successful AI application in pharmaceutical or biotech industries. Take, for example, the well known in the industry 'IBM's Watson Artificial Intelligence (AI) and Data Platform', which since 2010 integrates technologies and cloud-services to support cognitive businesses. Watson has strategic collaborations with three out of the five leading pharmaceutical companies. Analysts report that it is too early to see any meaningful effect of IBM's technology on either patient outcomes or on specific goals of each company-partner. ${ }^{23}$

Digital disruption and augmentation of pharma R\&D with artificial intelligence (AI) is well defined in the phenomenal report by EY/VitalSigns, ${ }^{21}$ where term AI is frequently used to describe just the machine learning or deep learning algorithms. As such, this type of technology is currently employed to develop tools for global research collaborations, customer engagement, innovative models for partnership support, and secure data management in biomedical research. Current initiatives are primarily focused on leveraging AI solutions for chronic disease management in the areas of cancer and diabetes. Understandably, the concerns about privacy, data protection and governance keep growing as novel technologies expand onto healthcare sectors. ${ }^{24}$

The Technology strategy will tackle appropriately an avalanche of advances in drug discovery and development pipeline (e.g. genetic and bioengineering technologies), the data deluge and silos from Internet, $\mathrm{R} \& \mathrm{D}$, automated production processes, and more. However, advances in Technology will not directly increase Pharma profits, “... digital technologies are about limiting the impact of price reductions, maintaining formulary positioning and generating competitive differentiation...", says Lisa Suennen, Senior Managing Director at the GE Ventures.

\section{Conclusion}

This summary of projections and trends in pharmaceutical industry for 2017-2018 confirms the notion that the ongoing complex transformation of the industry requires a fresh look at business models and strategies. New genetic, bio-engineering, and digital technologies continue to converge synergistically while creating novel solutions and services that narrow the gap between health-span (disease free lifetime) and life-span so that individuals can live better for longer. We live in the exciting time of changes in Pharmaceutical industry that will bring new hope and better treatments to patients, to each of us. Innovative design of corporate strategy with special attention to Technologies will revolutionize drug development industry with outlook for the near future.

\section{Acknowledgements}

The author would like to express her sincere gratitude to Dr. Louise Bergeron, VP of Biology at Encycle Therapeutics, Canada, for inspiration and valuable insights about the drug development industry. This publication would not be possible without the team of experts at Vesti Intelligence Corp., USA, who contributed generously by discussing the topic and providing their guidance. Lastly, special thanks go to author's family for being her biggest supporter.

\section{Conflict of interest}

The author declares there is no conflict of interest.

\section{References}

1. Global Pharma Market Will Reach \$1.12 Trillion In 2022 Pharmaceutical Commerce website. 2016.

2. Pratt C. Top Pharmaceutical Trends for 2017. Pharmaceutical Investing News website. 2017.

3. Pisani J, Lee MA. Critical Makeover For Pharmaceutical Companies: Overcoming Industry Obstacles With A Cross-Functional Strategy. PWC Strategy\& website. 2017.

4. Soelkner P. Past, Present, and Future Trends Affecting the Bio/ Pharmaceutical Industry. Pharmtech website. 2017

5. Bigelow P, Calitri C, Leonard S, et al. Looking Ahead - 2017, Pharmaceutical Industry Trends. ISPE Connecting Pharmaceutical Knowledge website. 2017.

6. Price Water House Coopers \& PWC report. Pharma 2020: The Vision. Which Path Will You Take? 2007.

7. Arlington S, Palo J, Davies N, et al. From Vision To Decision 2020, Pharma Life Sciences, Pharma 2020. PWC website. 2012.

8. Loffler A, Stern S. The Future of the Biomedical Industry In An Era Of Globalization. Kellogg School of Management, Northwestern University. USA; 2007.

9. Esposito RS, Ostro MJ. Strategic consolidation: the biotechnology business model for the $21 \mathrm{st}$ century. $J$ Nature Biotechnology. 1999; $17: 10052317$. 
10. Ng A. Deep Learning's Next Frontier. Harvard Business Review webinar website. 2017.

11. Office for Official Publications of the European Communities. Life Sciences and Biotechnology - A Strategy For Europe. Luxembourg. 2002: pp 25-46.

12. Guerin S. Obamacare to Trumpcare-Impact on Pharma. 2017.

13. Penrice T. 7 Top Pharma Trends for 2017. Pennside Partners website. 2017.

14. Seven Pharma Trends for 2017. Pharmafile website. 2016.

15. Mesko B. 6 Surprising Trends Shaping the Future of Pharma. Medicalfuturist website. 2016.

16. Van Arnum P. Outlook 2018: The Current and Future Direction of the Pharma Industry. DCAT Value Chain Insights website. 2017.

17. Acton A. 5 Important Healthcare predictions for 2018. Forbes website. 2017.
18. Iervolino A, Urquhart L. World Preview 2017, Outlook to 2022. Evaluate Pharma website. 2017.

19. Murphy S, Patricola L, Baudouin N, et al. Industry Top Trends 2018: Health Care. S\&P Global Ratings website. 2017.

20. Meiling B. Six Top Biotech Vcs Take a Look at the Latest Trends, and Offer Their Thoughts on 2018. Endpoint News website. 2017.

21. Giovannetti GT, Spence P. Biotechnology Report 2017. Beyond borders, staying the course. Ernst \& Young Global Limited website. 2017.

22. Recent HIPAA Changes. HIPAA Journal website. 2018.

23. Sennaar K. AI in Pharma and Biomedicine - Analysis of the Top 5 Global Drug Companies. Tech Emergence website. 2018.

24. Sengupta S. Machine learning and artificial intelligence (ai) in biotechnology - we are in a golden age of medical research. BioStorage Technology website. 2017. 